\title{
Deep-gray nuclei susceptibility-weighted imaging filtered phase shift in patients with Wilson's disease
}

\author{
Xue Bai', Guangbin Wang' ${ }^{1}$ Lebin Wu' ${ }^{1}$ Yubo Liu' ${ }^{2}$ Li Cui ${ }^{3}$, Honglu Shi' and Lingfei Guo'
}

BACKGROUND: Susceptibility-weighted imaging (SWI) is a useful tool for evaluating brain paramagnetic mineralization. The aim of this study was to evaluate SWI filtered phase shift in brain gray nuclei of Wilson's disease (WD).

METHODS: Twenty-three WD patients and 23 age- and gender-matched healthy controls underwent SWI. Phase values of bilateral brain gray nuclei were measured on corrected phase image of all subjects.

RESULTS: Compared with healthy controls, WD patients showed a trend of negative phase shift in all regions of interest, and significantly lower phase value was found in bilateral putamen (PU) (left $P=0.009$, right $P=0.001$ ), caudate (left $P=0.001$, right $P=0.001$ ), thalamus (TH) (left $P<0.001$, right $P<0.001$ ), red nucleus (left $P=0.031$, right $P=0.049$ ), and substantia nigra (left $P=0.003$, right $P=0.047$ ). The WD patients groups were divided into neurological, hepatic, and asymptomatic onset subgroups. And neurological onset patients had lower phase value than hepatic onset patients on bilateral PU (left $P=0.025$, right $P=0.002$ ) and TH (left $P=0.025$, right $P=0.025$ ).

CONCLUSION: Abnormal negative phase value was significantly increased in brain gray nuclei of WD patients, giving evidence in vivo about paramagnetic mineralization accumulating in brain gray nuclei. The phase shift of SWI could be used as a potential biomarker to help in diagnosing and evaluating WD.

W ilson's disease (WD), known as hepatolenticular degeneration, is an autosomal recessive inherited disorder of copper metabolism. It is characterized by an excretion dysfunction of copper, leading to the toxic deposition in the liver and the brain, causing chronic liver disease or/and progressive neurologic and psychiatric impairment. Liver and brain biopsy have demonstrated copper deposition of WD in liver and brain $(1,2)$. Animal model studies have also indicated that copper and iron accumulate in brain striatum and substantia nigra (SN) $(3,4)$. Magnetic resonance imaging (MRI) is a useful tool for diagnosis of various neurometabolic diseases, and has been used to diagnose and evaluate prognosis of WD. It is characterized with T2-weighed imaging (T2WI) and T2 weighted fluid attenuated inversion recovery (T2-FLAIR) hyperintense lesions, T1-weighted imaging (T1WI) hypointense lesions involving bilateral lenticular nucleus, caudate (CA) nucleus, and/or thalami, midbrain, with "face of the giant panda" sign in the midbrain and "face of panda cub" sign in the pons $(5,6)$. Although Andersen et al. have reported the abnormal high signal on T1WI and low signal on T2WI in MR imaging of WD patients, speculating the probability of paramagnetic effect of the copper accumulation (7). However, the high-signals on T2WI in brain gray nuclei, which represent pathological changes, such as edema, gliosis, neuronal necrosis, or spongiform degeneration $(8,9)$, usually cover up the paramagnetic T2 hypointense. Thus, it is difficult for traditional MR imaging to evaluate the brain mineral deposition in WD patients.

Recently, improved MRI technique, such as susceptibilityweighted imaging (SWI), which provides unique contrastbased on tissue susceptibility differences, has been shown to be superior in its ability to demonstrate paramagnetic substance deposition in brain (10). Several previous studies have indicated that SWI phase shifts correlate with brain iron concentration in various brain regions of healthy adults (11). Copper is also paramagnetic ion (12). In physiological phenomenon, the concentrations of brain copper are too small to produce a detectable MR contrast. Some studies demonstrated that under pathological conditions, it is conceivable that in some regions brain copper content could be sufficient to be detectable by MR imaging (13). In this study, we utilized the measurement of corrected phase images based on SWI to investigate whether there were phase shifts between WD patients and healthy controls in brain gray nuclei and whether symptoms related with phase value.

\section{RESULTS}

\section{Population Data}

The demographic and laboratory parameters of all the subjects were demonstrated in Table 1. A total of 23 WD-diagnosed patients were evaluated, including 15 males and 8 females, the mean age was $17.1 \pm 8.7 \mathrm{y}$ (range 6-35 y). At the time of evaluation with MRI, the mean duration of onset was $8.3 \pm 10.8 \mathrm{mo}$. The mean values of the blood ceruloplasmin, blood copper and 
Table 1. Clinical characteristics, laboratory parameters, and phase values of 23 WD patients and HC

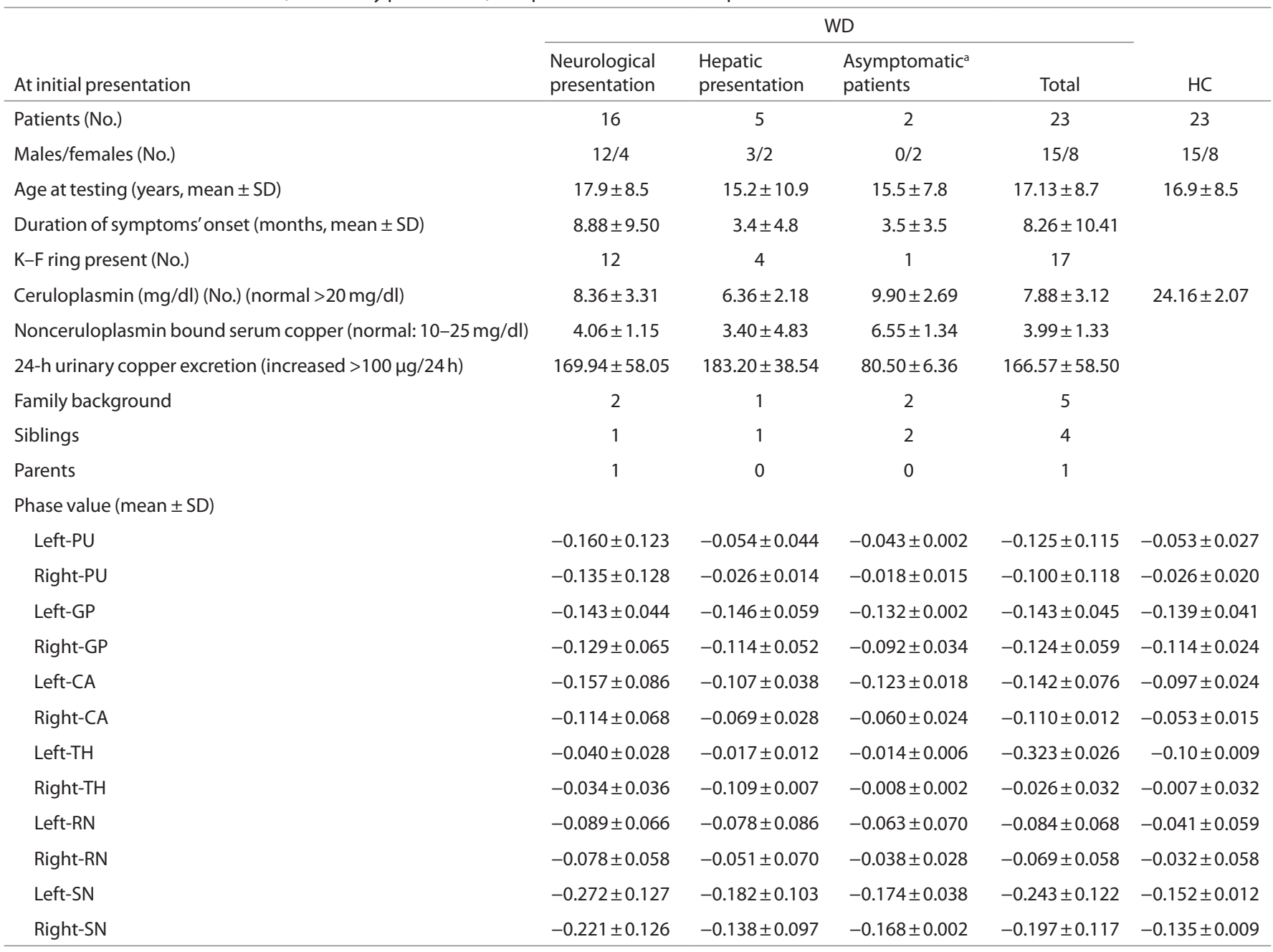

aThe asymptomatic patients were identified due to family screening. The two recruited asymptomatic patients were siblings of one neurological WD patient and one hepatic WD patient, respectively.

CA, caudate; GP, globus pallidus; HC, healthy controls; K-F ring, Kayser-Fleischer ring; No., number; PU, putamen; RN, red nucleus; SN, substantia nigra; TH, thalamus; WD, Wilson's disease.

urinary copper were $7.88 \pm 3.12 \mathrm{mg} / \mathrm{dl}, 3.99 \pm 1.33 \mathrm{mg} / \mathrm{dl}$, and $166.57 \pm 58.50 \mu \mathrm{g} / 24 \mathrm{~h}$, as well as a presence of a Kayser-Fleischer (K-F) ring in 17 patients (73.9\%). Of these 23 patients, 19 patients (82.6\%) were newly diagnosed with $\mathrm{WD}$, and the other 4 patients (17.4\%) already received medical therapy, such as D-penicillamine $(n=4)$, or/and zinc $(n=1)$ at the time of MRI.

In order to study the clinical manifestations, we divided symptoms or signs into hepatic, neurological, and asymptomatic groups. Sixteen of 23 patients (69.57\%) had onset with neurological manifestations and $5(21.74 \%)$ with hepatic manifestations. Two patients $(8.70 \%)$ were "asymptomatic" at the time of diagnosis and were identified on family screening. The most frequent symptoms in our WD patient population were of neurological origin in more than half of case. The patients were from $21 \mathrm{fami-}$ lies, 2 asymptomatic patients were siblings of one neurologic symptom patient and one hepatic symptom patient, respectively.

Conventional brain MRI was abnormal in all $16(100 \%)$ neurologic origin patients, $3(60 \%)$ hepatic origin patients, and was normal in $2(100 \%)$ asymptomatic patients. Most of abnormalities in basal ganglia were typical symmetrical or asymmetrical hypointense on T1WI (Figure 1a) and hyperintense on T2WI (Figure 1b), with 11 patients having hypointense lesions on T2WI (Figure 1f). No patients showed hyperintense lesion on T1WI. While all the 17 patients with abnormal imagings have sharp margined hypointense lesions on SWI (Figure 1,h).

\section{Phase Shift Between WD Patients and Healthy Controls}

To assess the phase shift between WD patients and healthy subjects, we analyzed all the $23 \mathrm{WD}$ patients recruited and 23 age- and sex-matched healthy controls. The results showed all the phase value data measured from regions of interests of WD patients were lower than those of control subjects (Table 2, Figure 2), and significantly negative phase shift was found in bilateral putamen (PU) (left $P=0.009$, right $P=0.001$ ), CA (left $P=0.001$, right $P=0.001$ ), thalamus (TH) (left $P<$ 0.001 , right $P<0.001$ ), red nucleus (RN) (left $P=0.031$, right $P=0.049$ ) and SN (left $P=0.003$, right $P=0.047$ ) (Figure 2). 


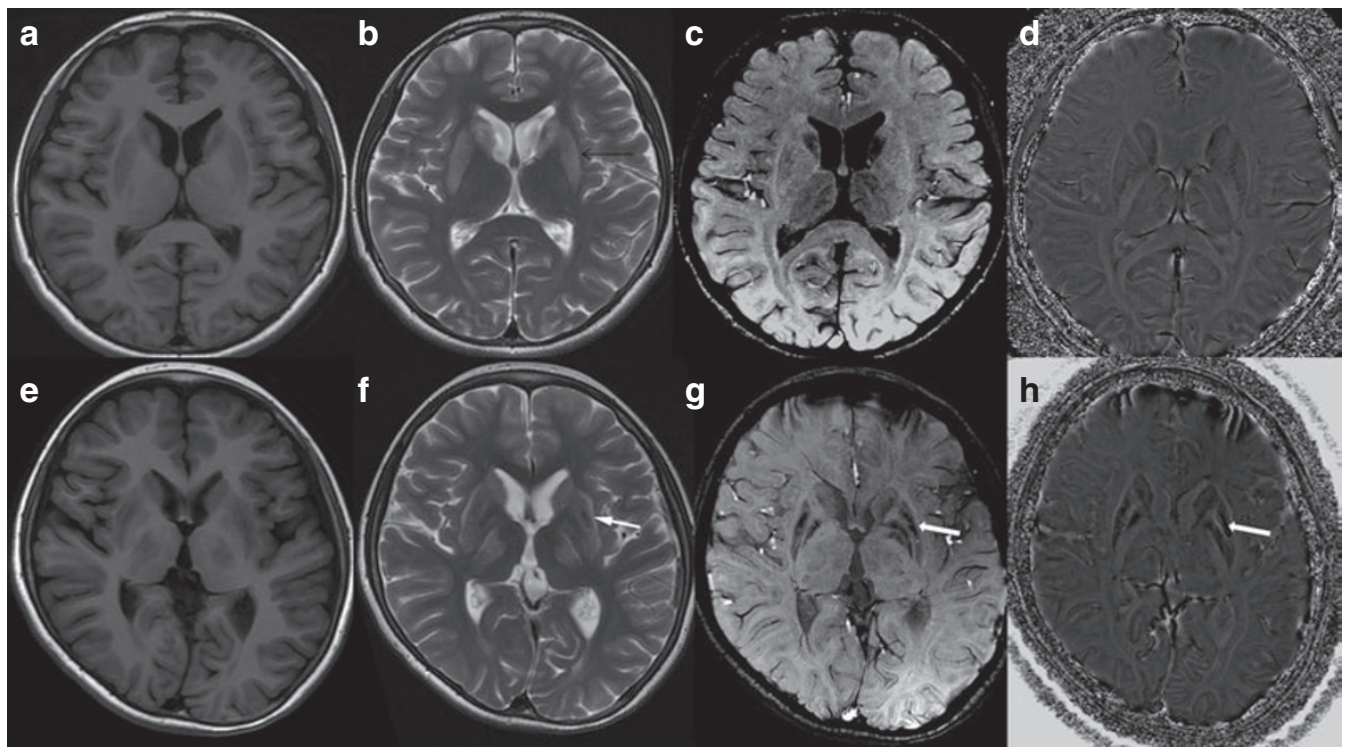

Figure 1. Illustration of magnetic resonance imaging and susceptibility-weighted imaging (SWI) characteristics of Wilson's disease patients. (a-d) T1WI, T2WI, SWI corrected magnetic image and corrected phase image of a $12 \mathrm{y}$ old diagnosed Wilson's disease (WD) girl; (a,b) the T1WI hypointense and T2WI hyperintense on bilateral putamen (dark arrow) and caudate, which indicate the pathological process of edema, gliosis, neuronal necrosis, or spongiform degeneration. (e-h) T1WI, T2WI, corrected magnetic image and corrected phase image of a 11-y-old female patient; (f) nodular hypointense lesion on left putamen (white arrow), indicating paramagnetic signals; ( $\mathbf{g}, \mathbf{h})$ corrected magnetic image and corrected phase image, showing marked margin of dark lesion on left putamen (white arrow).

Table 2. Phase values ${ }^{\mathrm{a}}$ for each $\mathrm{ROI}$ of all participants

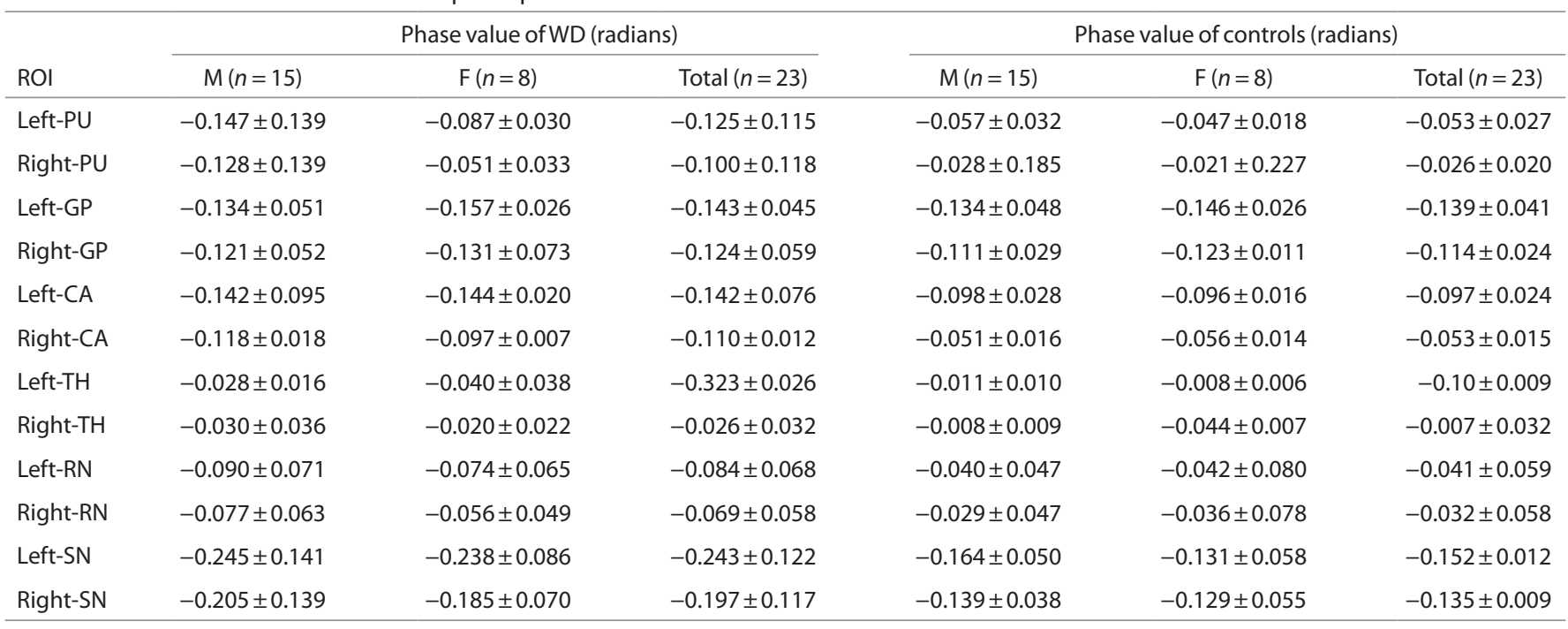

CA, caudate; F, female subjects; GP, globus pallidus; $M$, male subjects; $P U$, putamen; RN, red nucleus; $R O I$, region of interest; $S N$, substantia nigra; $T H$, thalamus.

aphase value $=$ mean + SD.

There is no significant difference in left and right globus pallidus (GP) (left $P=0.372$, right $P=0.130$ ).

\section{Phase Shift Between Different Symptom Groups}

Among the 23 patients with WD, 16 patients originated with neurological manifestations, 5 with hepatic manifestations. In order to probe whether different symptoms related with different phase values in different regions, we analyzed our data according to different symptoms. The results showed that, in most of brain region measured, the phase value of neurological WD patients were higher than that of hepatic WD patients (Table 1, Figure 3), and there was significant lower negative phase value in bilateral PU (left, $P=0.025$; right, $P=0.002$ ) and bilateral TH (left, $P=0.025$; right, $P=0.025$ ) of neurological WD patients, compared with hepatic WD patients.

\section{DISCUSSION}

SWI is a high-resolution, 3D fully flow-compensated gradient echo sequence, it uses magnitude and phase information, to enhance the local tissue susceptibility variations information (14). Its brilliant phase shift at high magnetic fields has been well documented, in particular for mineral storage. Phase images contain numerous information about susceptibility diversity among tissues, which can be useful in evaluating 


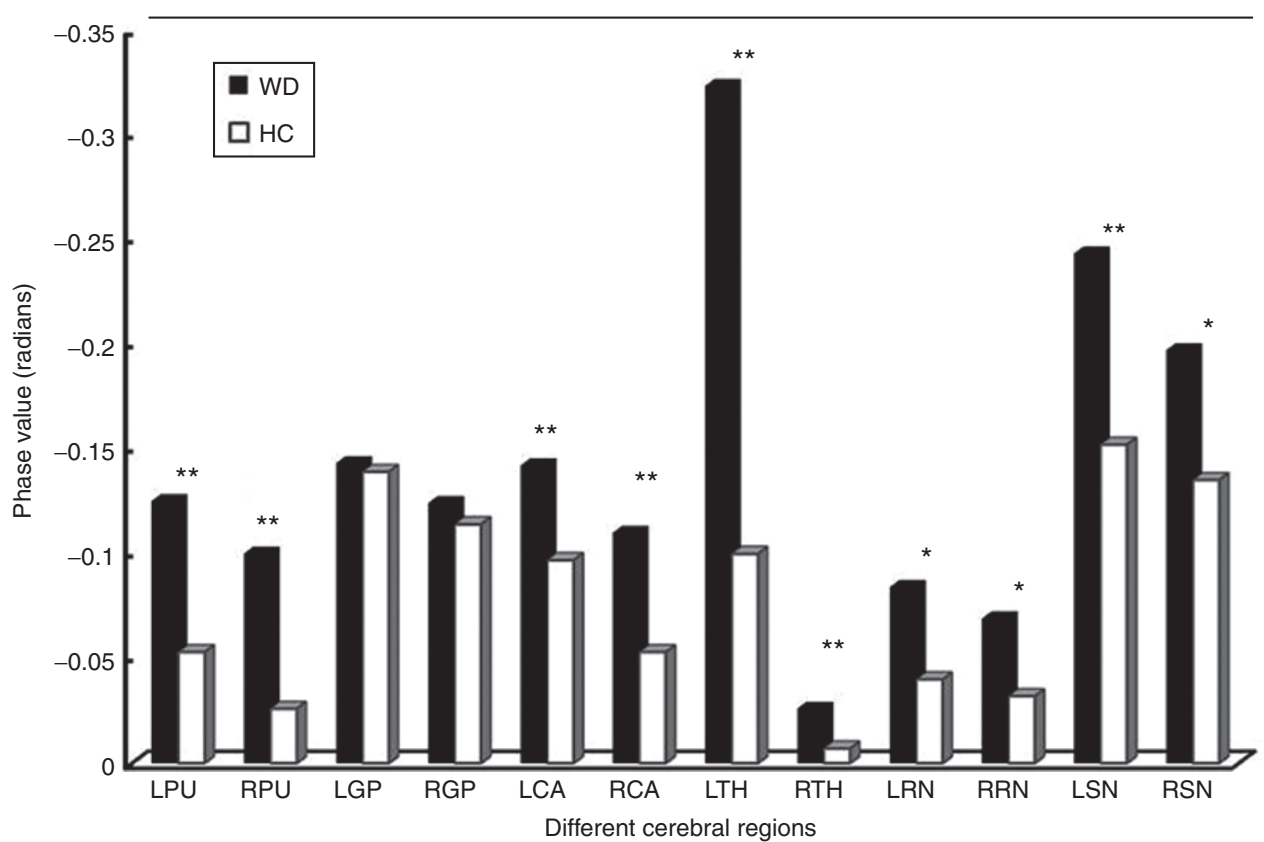

Figure 2. Phase shift of bilateral regions of interest (ROIs) between Wilson's disease (WD) and healthy controls (HC). Black column corresponds to the mean phase value of WD patient $(n=23)$ and white column corresponds to the mean phase value of healthy controls $(n=23)$. Differences of phase shift of ROIs between WD and healthy controls are analyzed using Mann-Whitney $U$-tests, and significant differences are indicated with ${ }^{*}(P<0.05)$, ${ }^{* *}(P<0.005)$.

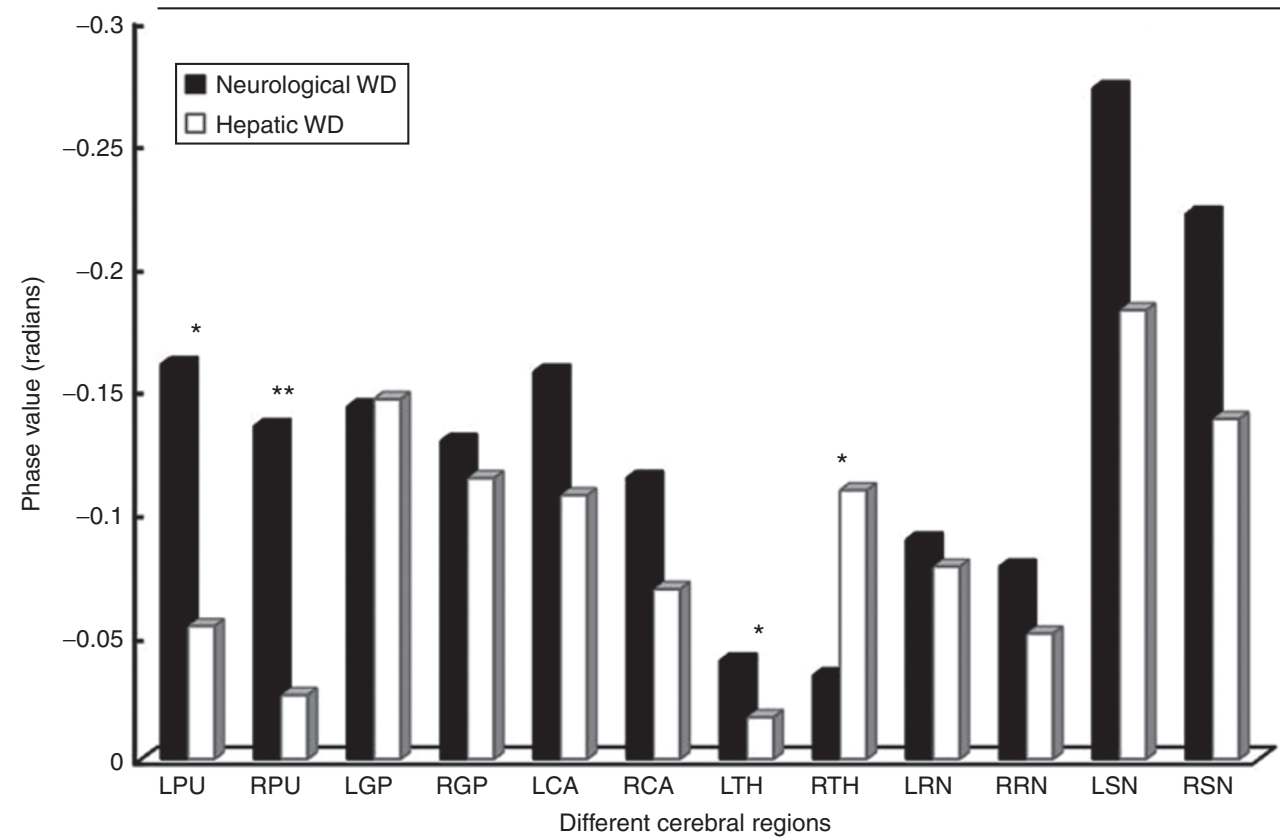

Figure 3. Phase value difference between neurological Wilson's disease (WD) patients and hepatic WD patients. Black column corresponds to the mean phase value of neurological WD patients $(n=16)$ and white column corresponds to the mean phase value of hepatic WD patients $(n=5)$. Differences of phase shift of regions of interest (ROIs) between WD and healthy controls are analyzed using Mann-Whitney $U$-tests, and significant differences are indicated with * $(P<0.05){ }^{* *}(P<0.005)$.

mineralization that changes the local field (15), including paramagnetic substance and diamagnetic substance.

\section{Decreased Phase Value in Gray Nuclei of WD Patients}

The results showed there was lower phase value in brain gray nuclei of WD patients, relative to healthy controls. Significantly decreased phase value was detected in bilateral PU, CA, TH, RN, and SN, compared with age- and gender-matched healthy controls. Studies have confirmed the negative relationship between paramagnetic mineralization and phase value $(16,17)$, so the negative phase shift of our study indicated that there is paramagnetic mineralization primarily depositing in these regions.

Copper is paramagnetic ion. In physiological phenomenon the concentrations of copper in the brain are too small 
to produce a detectable MR contrast. Some studies demonstrated that under pathological and experimental conditions, it is conceivable that brain copper content could be sufficient to be detectable in specific regions using MR imaging (2). Furthermore, WD is a copper overloading disease, brain copper concentration has been reported to be 10 - to 15 -fold than in normal conditions (reference values are 13 to $60 \mathrm{mg} / \mathrm{g}$ ). Autopsy studies have reported that the mean concentration of brain copper, ranging from 88 to $158 \mu \mathrm{g} / \mathrm{g}$ of dry tissue, is higher than normal reference value $(2,18)$. Moreover, remarkably increased copper level has been found in rat model of WD (4). Therefore, it is speculated that the negative phase shift mostly reflexes copper deposition. However the contribution of copper to the degree of susceptibility effects in SWI has not been determined yet.

Previous studies have focused most on the relationship between SWI and iron level, and proved that SWI phase shifts correlated with brain iron concentration $(11,19)$. WD is a ceruloplasmin deficient neurodegenerative disease, and ceruloplasmin plays an important role in iron release from the brain neuronal cells (20). Ceruloplasmin is considered as the critical ferroxidase, and it plays a role in oxidizing the ferrous iron $\left(\mathrm{Fe}^{2+}\right)$ to ferric iron $\left(\mathrm{Fe}^{3+}\right)$. Reduced Cp ferroxidase activity could lead to cellular iron overload, and subsequently, to elevated ferritin level. When serum ceruloplasmin decreased to extremely low level, iron abnormality may come into being in WD (21). Iron deposition in brain gray nuclei had been demonstrated in previous animal model study (3). Iron, compared with copper, is powerful paramagnetic substance. Then the negative phase shift may be much more contribute to the paramagnetic effete of iron which excessive the effect induced by copper accumulation.

In the absence of comparative neuropathologic MRI studies, the nature of dark-signal lesions remains unclear. The excessive copper and iron has been found both existing in WD rat brain (3). One more possibility is that the negative phase shift may be the result of copper and iron interaction.

\section{The Phase Shift of Bilateral GP}

Bilateral phase value in brain GP with WD had no difference with control groups, even though there was a trend of negative phase shift. One possible explanation may resolve our findings. Postmortem and in vivo studies have identified the GP being the most iron-rich structure (up to $200 \mu \mathrm{g} / \mathrm{g}$ tissue wet weight) regardless of age $(22,23)$, the iron concentration of GP is already in high level in healthy brain. The degree of copper accumulation, with or without iron elevation, in GP might be too small to induce statistical phase shift. Furthermore, iron is super-paramagnetic substance, the phase shift induced by iron would cover up that induced by copper. Therefore, although the phase value of bilateral GP decreased in WD patients, there is no statistical difference.

\section{The Clinical Manifestations and Phase Shift}

WD is a disorder of copper dysbolism, absent or reduced function of ceruloplasmin leads to decreased hepatocellular excretion of copper into bile. This results in hepatic copper accumulation and leading to hepatic injury and symptoms (24). Also, the overload of brain copper impairs the neurons and results in neurological disorder (25). It is considered that accumulation of copper occurs early in liver (26), and hepatic symptoms are the most common onset of WD, so when the neurologic symptoms occur, more copper has deposited in brain regions than that with mere hepatic symptoms. In our study, the higher negative phase value in neurologic WD patients in brain regions support this pathological process.

\section{Limitations and Future Work}

In this study, the nature of negative phase shift is mostly based on literatures inference. Whether copper or iron mainly contribute to the phase shift, neuropathology study would be needed to make it clear in the future study. Furthermore, copper is mild paramagnetic ion, a paucity of studies have investigated the effect of copper on the SWI, and the contribution of copper to the degree of phase shift in SWI has not been determined yet. All of these are also necessary work to make it clear in the future study. Furthermore, the sample size in this study is small. There are only two asymptomatic WD patients were recruited, which is too small to be analyzed, so in the future study, we will continue the increase of subjects sample in order to complete the study on evaluating the paramagnetic ion accumulation among different symptoms of WD.

\section{Conclusion}

In this study, the negative phase shift is detected in WD brain gray nuclei on SWI, in both symptomatic groups and asymptomatic group, suggesting there was paramagnetic ion deposition in vivo. Moreover, the lower negative phase value on neurological onset patients indicates more paramagnetic ion deposition than hepatic onset patients. These results imply that the phase shift of SWI could be used as a potential biomarker to help diagnosing and evaluating WD.

\section{METHODS}

\section{Subjects}

Twenty-three patients diagnosed of WD (15 males and 8 females, range $=6-35 \mathrm{y}$, mean $=17.1 \pm 8.7 \mathrm{y}$ ) and twenty-three age- and gender-matched healthy controls were evaluated. Sixteen patients were neurological onset, five with hepatic onset, and two with no symptoms. All the WD patients were diagnosed basing on their clinical examinations, laboratory and imaging findings. All the age- and gender-matched control subjects, with normal blood ceruloplasmin, had no history of neurologic/psychiatric disorders, neither hepatic disease, abnormal signal in structural MRI. Two patients were excluded because of poor quality imaging.

This study was approved by Shandong University ethics board, all subjects or their legal representatives gave informed consent for participation.

\section{MRI Protocol}

All the MRI examinations were performed on a 3.0-T system (Signa HDx, GE Medical System, Milwaukee, WI) equipped with a standard eight-channel head coil. The head was immobilized in the head coil with foam padding without any left-right tilt. The imaging protocol included: sagittal T2-weighted (T2W) fast spin-echo (FSE) (TR/TE 4500/122), axial T1-weighted fluid attenuated inversion recovery (T1-FLAIR) (TR/TE 1957/27), T2-weighted fluid attenuated 


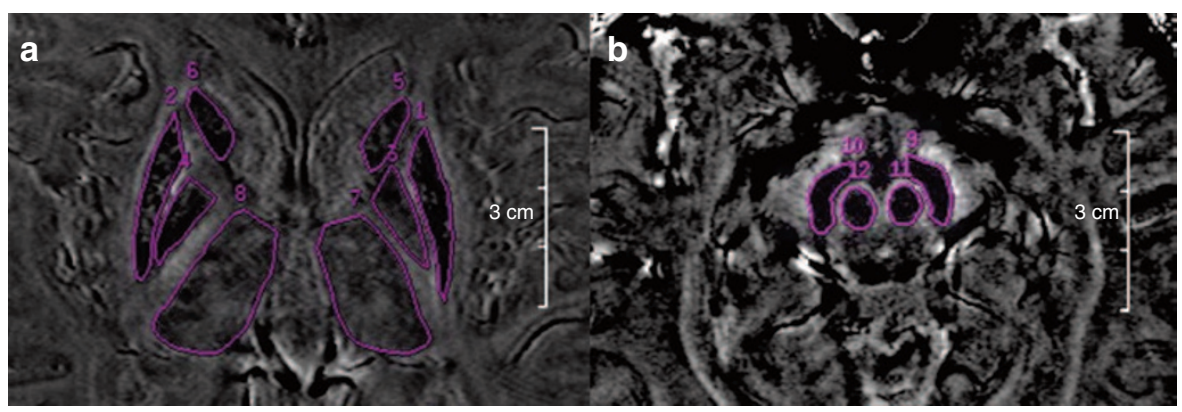

Figure 4. Display of the (a) basal ganglia and (b) the midbrain of a patient with Wilson's disease (WD), drawing 12 gray matter areas as representative regions of interest with a magnification factor of 2.0. 1, $2=$ bilateral PT; $3,4=$ bilateral GP; $5,6=$ bilateral CA; 7, $8=$ bilateral TH; $9,10=$ bilateral SN; $11,12=$ bilateral RN.

inversion recovery (T2-FLAIR) (TR/TE 9002/157), with field of view $24 \mathrm{~cm} \times 24 \mathrm{~cm}, 256 \times 320$ matrix, $5 \mathrm{~mm}$ slices thickness, $1.5 \mathrm{~mm}$ slice gap. Sagittal T2-FSE images were acquired to locate the exact positions of the anterior and posterior commissural line (AC-PC line). Routine magnetic resonance scanning range covered the whole brain. The susceptibility-weighted images were performed parallel to the $\mathrm{AC}-\mathrm{PC}$ line and covered the nuclei of the basal ganglia and mesencephalon. The protocols were set as follows: repetition time (TR) = $38 \mathrm{~ms}$, echo time $(\mathrm{TE})=25 \mathrm{~ms}$, flip angle $=20^{\circ}$, matrix $=448 \times 256$ $(\mathrm{Nx} \times \mathrm{Ny})$, field of view $=24 \mathrm{~cm} \times 24 \mathrm{~cm}$. A total of 50 slices were collected with a slice thickness of $2.0 \mathrm{~mm}$ and no gap between slices. Both original phase and magnitude images were acquired, only phase data were used for further analysis in this study.

\section{Image Processing}

All the data processing were operated on a separate workstation (ADW4.3, GE), phase images were high-pass filtered to create corrected phase images with a central matrix size of $64 \times 64$ (15), in order to remove varying phase shifts which arise predominantly from background field inhomogeneities and air-tissue interfaces $(15,27)$. Twelve structures including bilateral PU, GP, CA, TH, RN, and SN were measured on the corrected phase images, and the phase value ranged from $-\pi$ to $+\pi$.

The regions of interest were identified and hand-drawn according to the anatomical structures by a trained neuroradiologist, blinded to subject data. The value showed on the corrected phase image was the mean phase value of the structure drawn. The structures were zoomed-in by a factor of 2.0 to make the boundaries easier to define. The first, second and third slices above the AC-PC line were used to obtain data from the bilateral GP, PU, CA, TH (Figure 4a), and the final values were the means of the drawn. The data of the SN and $\mathrm{RN}$ were obtained from the slice in which the region of interest was largest (Figure 4b), which demonstrated the most well-defined border, and represented the expected area for each interested region. Furthermore, the slices which were severely affected by sinus artifacts were excluded.

\section{Statistics}

All statistical analyses were performed by operators blinded to participants' status with the use of Statistical Package for Social Sciences version 17.0 software for Windows (SPSS, Chicago, IL). After the analysis about the normality of the data (Shapiro-Wilk test), the distributions were not normal $(P<0.05)$. Mann-Whitney $U$-tests were used to compare all the regions of interests phase values of WD patients with those of control subjects, and to evaluate whether there was phase difference between neurological origin patients and hepatic origin patients. Because there were only two asymptomatic patients, the sample size was too small; the comparison between symptomatic patients and asymptomatic patients was not evaluated. For all the results, statistical significance is determined as $P<0.05$.

\section{ACKNOWLEDGMENTS}

We are grateful for the support of E. Mark Haacke for entitling us to use SWI sequence on GE Medical System (Signa HDx, Milwaukee, WI). We are thankful to our patients who consented to the study.
Disclosure: The authors declare no conflict of interest.

\section{REFERENCES}

1. Ferenci P, Steindl-Munda P, Vogel W, et al. Diagnostic value of quantitative hepatic copper determination in patients with Wilson's Disease. Clin Gastroenterol Hepatol 2005;3:811-8.

2. Faa G, Lisci M, Caria MP, et al. Brain copper, iron, magnesium, zinc, calcium, sulfur and phosphorus storage in Wilson's disease. J Trace Elem Med Biol 2001;15:155-60.

3. Kim JM, Ko SB, Kwon SJ, et al. Ferrous and ferric iron accumulates in the brain of aged Long-Evans Cinnamon rats, an animal model of Wilson's disease. Neurosci Lett 2005;382:143-7.

4. Hayashi M, Fuse S, Endoh D, et al. Accumulation of copper induces DNA strand breaks in brain cells of Long-Evans Cinnamon (LEC) rats, an animal model for human Wilson Disease. Exp Anim 2006;55:419-26.

5. Singh P, Ahluwalia A, Saggar K, Grewal CS. Wilson's disease: MRI features. J Pediatr Neurosci 2011;6:27-8.

6. Jacobs DA, Markowitz CE, Liebeskind DS, Galetta SL. The "double panda sign" in Wilson's disease. Neurology 2003;61:969.

7. Andersen K, Südmeyer M, Saleh A. [Cerebral imaging for Wilson disease]. Rofo 2007;179:225-33.

8. Kim TJ, Kim IO, Kim WS, et al. MR imaging of the brain in Wilson disease of childhood: findings before and after treatment with clinical correlation. AJNR Am J Neuroradiol 2006;27:1373-8.

9. da Costa Mdo D, Spitz M, Bacheschi LA, Leite CC, Lucato LT, Barbosa ER. Wilson's disease: two treatment modalities. Correlations to pretreatment and posttreatment brain MRI. Neuroradiology 2009;51:627-33.

10. Sehgal V, Delproposto Z, Haacke EM, et al. Clinical applications of neuroimaging with susceptibility-weighted imaging. J Magn Reson Imaging 2005;22:439-50.

11. Haacke EM, Ayaz M, Khan A, et al. Establishing a baseline phase behavior in magnetic resonance imaging to determine normal vs. abnormal iron content in the brain. J Magn Reson Imaging 2007;26:256-64.

12. Saini S, Frankel RB, Stark DD, Ferrucci JT Jr. Magnetism: a primer and review. AJR Am J Roentgenol 1988;150:735-43.

13. Schenck JF. Magnetic resonance imaging of brain iron. J Neurol Sci 2003;207:99-102.

14. Mittal S, Wu Z, Neelavalli J, Haacke EM. Susceptibility-weighted imaging: technical aspects and clinical applications, part 2. AJNR Am J Neuroradiol 2009;30:232-52.

15. Haacke EM, Xu Y, Cheng YC, Reichenbach JR. Susceptibility weighted imaging (SWI). Magn Reson Med 2004;52:612-8.

16. Zhu WZ, Zhong WD, Wang W, et al. Quantitative MR phase-corrected imaging to investigate increased brain iron deposition of patients with Alzheimer disease. Radiology 2009;253:497-504.

17. Jin L, Wang J, Zhao L, et al. Decreased serum ceruloplasmin levels characteristically aggravate nigral iron deposition in Parkinson's disease. Brain 2011;134(Pt 1):50-8.

18. Petrovecki V, Kovacic Z, Pizent A, Jakic-Razumovic J. [Copper levels in the tissues of patients with Wilson's disease]. Arh Hig Rada Toksikol 1997;48:241-6. 


\section{Articles Baietal.}

19. Sullivan EV, Adalsteinsson E, Rohlfing T, Pfefferbaum A. Relevance of Iron Deposition in Deep Gray Matter Brain Structures to Cognitive and Motor Performance in Healthy Elderly Men and Women: Exploratory Findings. Brain Imaging Behav 2009;3:167-75.

20. Harris ZL, Takahashi Y, Miyajima H, Serizawa M, MacGillivray RT, Gitlin JD. Aceruloplasminemia: molecular characterization of this disorder of iron metabolism. Proc Natl Acad Sci USA 1995;92:2539-43.

21. Ranganathan PN, Lu Y, Jiang L, Kim C, Collins JF. Serum ceruloplasmin protein expression and activity increases in iron-deficient rats and is further enhanced by higher dietary copper intake. Blood 2011;118:3146-53.

22. Hallgren B, Sourander P. The effect of age on the non-haemin iron in the human brain. J Neurochem 1958;3:41-51.
23. Bilgic B, Pfefferbaum A, Rohlfing T, Sullivan EV, Adalsteinsson E. MRI estimates of brain iron concentration in normal aging using quantitative susceptibility mapping. Neuroimage 2012;59:2625-35.

24. Roberts EA, Schilsky ML; American Association for Study of Liver Diseases (AASLD). Diagnosis and treatment of Wilson disease: an update. Hepatology 2008;47:2089-111.

25. Huster D. Wilson disease. Best Pract Res Clin Gastroenterol 2010;24:5319.

26. Sinha S, Taly AB, Ravishankar S, et al. Wilson's disease: cranial MRI observations and clinical correlation. Neuroradiology 2006;48:613-21.

27. Harder SL, Hopp KM, Ward H, Neglio H, Gitlin J, Kido D. Mineralization of the deep gray matter with age: a retrospective review with susceptibilityweighted MR imaging. AJNR Am J Neuroradiol 2008;29:176-83. 